\title{
Des capteurs chimiques à transduction optique pour mesurer la pollution de l'air intérieur
}

Gérer la pollution de l'air intérieur dans les établissements publics ou dans les habitats pour une meilleure qualité de vie deviendra dans le futur un concept normalisé de vie, tout du moins pour les pays les plus industrialisés. Les moyens pour y parvenir sont en bonne voie avec la volonté des pouvoirs publics de s'atteler sans tarder au problème de santé environnementale. Ils s'appuient également sur les avancées technologiques, notamment avec les capteurs chimiques à transduction optique, qui permettront un investissement à moindre coût d'appareillages de surveillance. Un exemple est ici exposé avec un capteur optique pour le formaldéhyde, un polluant ubiquiste de I'air intérieur, faisaint l'objet en France des réglementations en matière de qualité de l'air les plus avancées d'Europe.

Thu-Hoa TRAN-THI, Hélène PAOLACCI, Romain DAGNELIE, Sabine CRUNAIRE, Sandrine MARIANO, Wanxian WANG, Guillaume BRUNELLE,

Charles RIVRON, Laurent MUGHERLI, Emmanuel CHEVALLIER

CEA-Saclay, Laboratoire Francis Perrin, URA CEA-CNRS 2453

Yves BIGAY, Philippe KARPE, Sylvain COLOMB

Start-up Ethera

thu-hoa.tran-thi@cea.fr yves.bigay@ethera-labs.com

\section{La qualité de l'air intérieur}

\section{Un enjeu politique et socio-économique}

La thématique de la qualité de l'air intérieur est devenue un enjeu majeur dans la politique de santé environnementale. La pollution de l'air se caractérise par un ensemble de polluants d'origine chimique (composés organiques volatils, particules fines, oxydes d'azote ou de carbone) et biologique (moisissures, acariens). Elle touche l'ensemble de la population dont une grande partie passe en moyenne 80 à $85 \%$ de son temps dans un habitat fermé (domicile, transport, école ou lieu de travail, commerces, etc.). Or, selon la plupart des données toxicologiques et épidémiologiques, la pollution de l'air est notamment impliquée dans la genèse d'insuffisances respiratoires, de maladies cardiovasculaires, de I'asthme et de cancers. Depuis 2007, avec les Grenelles de I'Environnement et les Plans nationaux santé environnement (PNSE), les pouvoirs publics ont lancé de nombreuses actions visant à mieux connaître la composition de l'air intérieur aussi bien dans les lieux recevant du public que dans le milieu résidentiel. Ces actions sont menées avec l'aide des instituts et des agences françaises comme l'Observatoire de la Qualité de I'air intérieur (OQAI) [1], I'Institut national de l'environnement et des risques industriels (INERIS) [2], I'Agence française de sécurité sanitaire de l'environnement et du travail (AFSSET*) [3], le Laboratoire central de la surveillance de la qualité de l'air (LCSQA) et les Associations agréées de surveillance de la qualité de l'air (AASQA). Des bilans de ces campagnes de mesures, des valeurs guides de qualité de l'air intérieur ont été proposées pour les substances considérées comme prioritaires à surveiller [3] : formaldéhyde, monoxyde de carbone (CO), benzène, trichloroéthylène, particules de diamètre inférieur à $10 \mathrm{~m}$ (PM10), naphtalène, tétrachloroéthylène, phtalate de di(2-éthylhexyle) (DEHP), dioxyde d'azote, acétaldéhyde, ammoniac et radon.

\section{Les réglementations}

En s'appuyant sur les travaux de l'AFSSET, le Haut conseil de la santé publique (HCSP) a émis des avis sur des valeurs de

* L'AFSSET est devenu, depuis 2010, I'ANSES: Agence Nationale de Sécurité Sanitaire. gestion à suivre par les responsables des établissements recevant du public afin de les guider dans les actions à entreprendre en cas de dépassement de certains seuils dans les espaces clos.

Le premier polluant de l'air intérieur visé est le formaldéhyde, ubiquiste et classé cancérogène par le Centre international de recherche sur le cancer (CIRC) depuis 2004. Le HCSP a fixé quatre valeurs de gestion:

$-10 \mathrm{~g} / \mathrm{m}^{3}$ ou $8 \mathrm{ppb}$ (partie par milliard) : valeur cible à atteindre dans les dix ans ; $-30 \mathrm{~g} / \mathrm{m}^{3}$ ou $24 \mathrm{ppb}$ : valeur repère de la qualité de l'air en dessous de laquelle aucune mesure de gestion n'est à envisager ;

$-50 \mathrm{~g} / \mathrm{m}^{3}$ ou $40 \mathrm{ppb}$ : valeur d'information et de recommandation au-delà de laquelle il faudra dans un délai de quelques mois, identifier la ou les source(s) et mettre en œuvre les actions appropriées ;

- $100 \mathrm{~g} / \mathrm{m}^{3}$ ou $80 \mathrm{ppb}$ : valeur d'action rapide avec mise en œuvre d'une solution dans le mois suivant afin de redescendre à une concentration inférieure à $30 \mathrm{~g} / \mathrm{m}^{3}$.

Dans le cas des bâtiments neufs livrés à partir de 2012 ou de ceux faisant l'objet d'opérations de rénovation de grande ampleur, lesteneurs moyennes de $10 \mathrm{~g} / \mathrm{m}^{3}$, doivent être vérifiées avant la livraison aux occupants.

Par ailleurs, d'autres actions du PNSE 2008-2012 visent à la réglementation des matériaux ou produits émetteurs de 
formaldéhyde et à leur remplacement progressif. Les secteurs concernés sont nombreux, comme l'industrie de l'automobile, du bois, des plastiques, des colles, des textiles, des cosmétiques, les hôpitaux ou encore les morgues.

\section{Mesure du formaldéhyde : état de l'art}

Dans ce domaine de concentration faible, il n'existe pas d'appareil à faible coût permettant de mesurer directement et sélectivement le formaldéhyde. Le seul appareil sensible et sélectif est l'Aérolaser de AERO-LASER GmbH [4], dont la détection est basée sur la réaction de Hantzsch [5] de formation d'une lutidine à partir de la réaction sélective entre le formaldéhyde avec l'acétylacétone en présence d'une amine. Le produit fluorescent est détecté à $510 \mathrm{~nm}$ et la sensibilité annoncée est de 0,05 ppb. Le prix de l'appareil est cependant prohibitif (35 $k €$ ) et la maintenance est lourde du fait de la nécessité de réfrigérer les réactifs liquides. La méthode de référence (NF ISO 16000-3 pour l'échantillonnage actif et NF ISO 16000-4 pour l'échantillonnage passif), utilise comme réactif colorimétrique la 2,4-dinitrophénylhydrazine, qui réagit avec tous les aldéhydes et les cétones présents dans l'atmosphère à analyser pour former des hydrazones. Ces derniers sont séparés par chromatographie en phase liquide et analysés optiquement via leur spectre d'absorption. Cette méthode, bien que sélective, est lourde et coûteuse car elle nécessite une étape de prélèvement sur cartouche suivie d'une analyse en différé [6]. Les résultats sont la plupart du temps connus au plus tôt en deux semaines. Très récemment, Dräeger a mis sur le marché allemand un appareil destiné au grand public, le Biocheck, basé sur une méthode enzymatique sélective mais dont le seuil de détection reste élevé (50 ppb) [7].

Pouvoir fournir un appareil de mesure directe du formaldéhyde, à la fois sensible, sélectif, peu coûteux et facile d'utilisation reste donc un défi à relever. Dans ce domaine, les capteurs chimiques à base de matériaux nanoporeux et à transduction optique, alliant à la fois la fonction de concentration et une rapidité de réponse, apparaissent prometteurs.

\section{Les capteurs chimiques}

\section{Principe d'un capteur chimique}

Le principe d'un capteur chimique repose sur la reconnaissance moléculaire entre une molécule-sonde et un polluant-cible et sa transduction en un signal mesurable.

Le capteur chimique est donc constitué d'une couche sensible encapsulant des molécules-sonde ; les modes de transduction peuvent être électriques, électrochimiques, piézoélectriques ou encore optiques.

Pour l'élaboration de capteurs chimiques à la fois sensibles, sélectifs et rapides, la stratégie développée au laboratoire Francis Perrin repose sur l'utilisation :

- d'une couche sensible à base de silicate nanoporeux (film mince ou monolithe) à grande surface spécifique d'adsorption $\left(\sim 600 \mathrm{~m}^{2} \cdot \mathrm{g}^{-1}\right)$, de diamètre de pores adapté à celui du polluant-cible, et agissant comme une éponge pour concentrer ce dernier,

- d'une molécule-sonde, apte à réagir sélectivement avec le polluant-cible pour former un produit fortement coloré ou fluorescent,

- d'une méthode optique de détection (absorption ou fluorescence) pour une réponse rapide et directe avec un coût réduit grâce à l'utilisation de diodes électroluminescentes comme source de lumière.

\section{Capteur de formaldéhyde}

Dans le cas du formaldéhyde, la réaction très sélective choisie est celle du formaldéhyde avec le Fluoral-P produisant une molécule absorbant et fluoresçant dans le visible : le 3,5-diacétyl-1,4-dihydrolutidine (DDL) (fig. 1). Les molécules de Fluoral-P sont emprisonnées dans les pores d'un polymère inorganique, au cours d'une synthèse one pot où les réactifs (précurseurs silicés, solvant, eau) sont mélangés dans une même solution ou Sol. L'hydrolyse et la polycondensation des alcoxydes de silicium conduisent à la formation du gel, solide nanoporeux transparent.

Deux types de capteurs ont été réalisés. Le premier est un film mince $(200 \mathrm{~nm})$ du matériau dopé déposé par trempage sur un substrat en verre, et dédié à des mesures rapides de formaldéhyde par voie fluorimétrique. Un prototype de laboratoire a été mis au point, qui permet de suivre à la fois la variation d'absorbance et/ou de fluorescence des capteurs au cours de leur exposition à des teneurs calibrées de formaldéhyde (fig. 2). $\gg$

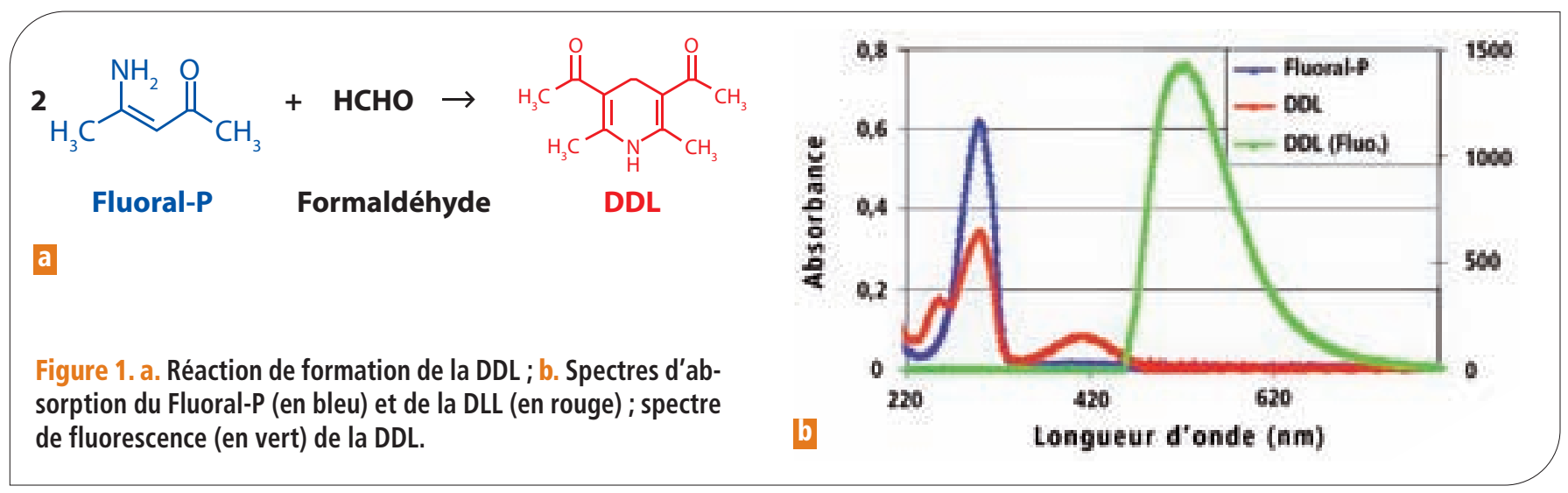




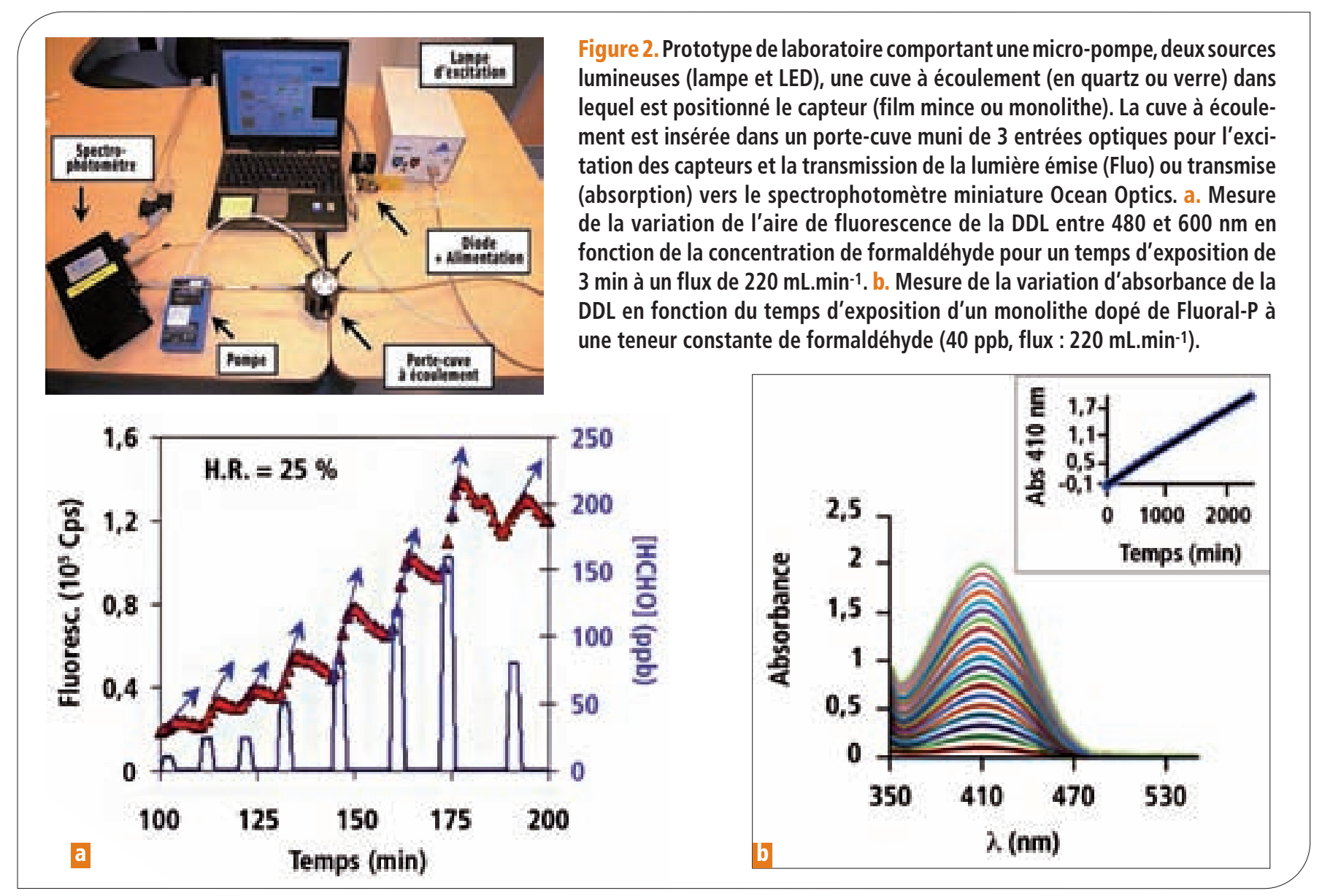

La fluorimétrie étant une méthode d'analyse particulièrement sensible, la sensibilité obtenue est élevée : 0,4 en ppb avec un temps de réponse de 15 minutes. Pour des teneurs plus élevées de l'ordre de la dizaine de ppb, ce temps est réduit à 2 à 3 minutes.

Le second capteur, dédié à la fois au grand public et aux professionnels, est un bloc monolithique de dimensions
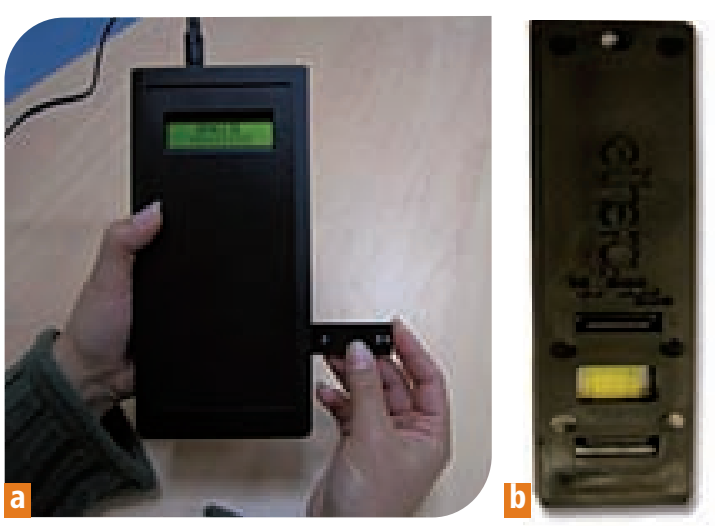

Figure 3. a. Introduction du capteur exposé au polluant dans le lecteur digital équipé d'une diode électroluminescente. b. Zoom sur le capteur dont la coloration jaune indique le piégeage de formaldéhyde. c. Nuancier de couleur pour une évaluation de la teneur en formaldéhyde en fonction de la durée d'exposition.

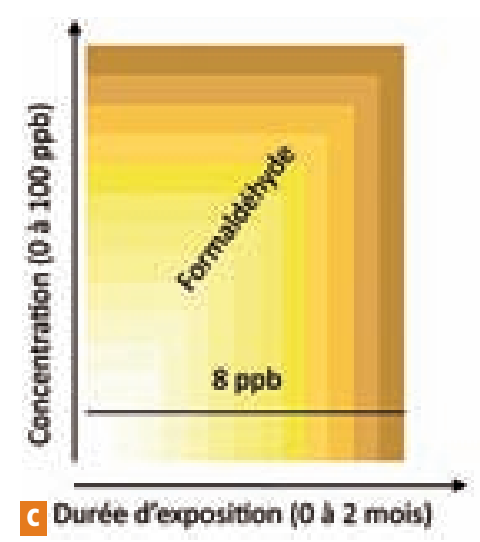

$8 \times 5 \times 2 \mathrm{~mm}$ (fig. 3). Le changement de couleur, de transparent à jaune, est suffisamment net pour une détection visuelle et une évaluation de la teneur de formaldéhyde grâce à un nuancier de couleur. Pour ce second capteur, un lecteur digital à coût modique, issu de la start-up Ethera, est associé pour une mesure plus précise de la teneur du polluant (fig. 3). La sensibilité obtenue est comme les capteurs de $\mathrm{O}_{2}$ (Presens
$\mathrm{GmbH}$ ) ou de $\mathrm{CO}_{2}$ et de $\mathrm{pH}$ (Gas Sensors

de 3 ppb pour une exposition en mode actif de $30 \mathrm{~min}$ à une vitesse de pom page de l'air contaminé de $220 \mathrm{~mL} \cdot \mathrm{min}^{-1}$.

Exposé de manière passive dans une pièce pendant plusieurs jours, il permet d'évaluer la teneur moyenne de formaldéhyde avec une précision de $20 \%$ pour des teneurs de quelques ppb. Exposé près d'une source émettrice de formaldéhyde comme un meuble en bois aggloméré ou un parquet en bois, le capteur permet de mesurer la quantité de formaldéhyde émise par le matériau-cible.

\section{Conclusion}

L'une des plus grandes difficultés pour l'industrialisation des matériaux Sol-Gel est de pouvoir reproduire de manière reproductible les différentes étapes de synthèse et de séchage qui conditionnent leur porosité. Ces difficultés sont plus aisément franchies lorsque ces maté- 
Solutions). Lorsque les matériaux sont sous forme de blocs monolithiques, la maîtrise de ces étapes devient primordiale pour l'obtention d'échantillons non craquelés et uniformément dopés.

Le capteur de formaldéhyde que nous venons de montrer est un exemple de transfert technologique en cours entre le laboratoire Francis Perrin et la startup Ethera, qui prend en compte toutes ces contraintes. Entre la mise au point des divers capteurs, films minces ou blocs monolithiques, et leur mise sur le marché, il aura fallu deux thèses [8 et 9], deux années de travail commun pour transformer un procédé de laboratoire en un procédé industrialisable et il faudra probablement à Ethera une année supplémentaire de mise au point pour le procédé industriel final. Ce n'est qu'à ce prix que des capteurs fiables pourront être mis sur le marché et concurrencer les appareils commercialisés.

Les capteurs de la start-up Ethera sont actuellement utilisés pour une campagne de mesures du formaldéhyde dans les écoles, menée par l'INERIS pendant I'an- née scolaire 2010-2011et dont les résultats seront rendus publiques en 2012. L'ingénierie des pores et la possibilité de réaliser des réactions chimiques dans les milieux confinés ouvrent la voie à de nombreux développements. D'autres capteurs pour l'air intérieur sont en cours de développement au laboratoire Fran-

\section{Références}

[1] S. Kirchner, J-F. Arenesi, C. Cochet, M. Derbez, C. Duboudin, P. Elias, A. Gregoire, B. Jédor, J-P. Lucas, N. Pasquier, M. Pigneret, O. Ramalho, Campagne nationale Logements. Etat de la qualité de l'air dans les logements français - Rapport final DDD/SB - 2006-57. Observatoire de la Qualité de l'Air Intérieur, 2006.

[2] J. Larbre, C. Marchand, Air intérieur. Bilan/veille sur la qualité de l'air intérieur à un niveau national et international : travaux récents et nouveaux instruments disponibles, rapport de l'INERIS, Dec. 2009.

[3] Guide de la qualité de l'air intérieur, VGAI : www. afsset.fr/index.php?pageid=829\&parentid=424

[4] www.aero-laser.de/AL4021IE.pdf

[5] T. Nash, The colorimetric estimation of formaldehyde by means of the Hantzsch reaction, The Biochemical Journal, 1953, 55(3), 416-421. cis Perrin. Ils concernent la détection des deux familles de polluants les plus présents dans les habitats, tels que les aldéhydes totaux ou les hydrocarbures aromatiques totaux, mais aussi d'autres polluants présents dans les lieux publics tels que le chlore et les chloramines dans les piscines couvertes.

[6] www.lcsqa.org/thematique/missions-diverses/airinterieur/elaboration-de-protocolesde-surveillancedu-formaldehyde

[7] www.draeger.com/FR/fr/products/gas_detection/ indoor_air/com_bio-check_formaldehyde.jsp

[8] H. Paolacci, Capteurs Chimiques à Transduction Optique de Polluants Atmosphériques à base de Matériaux Nanoporeux. Application au Formaldéhyde et autres Composés Carbonylés, Thèse, 2006, Université de Paris XI.

[9] R. Dagnelie, Thèse, Développement d'un Capteur Chimique de PolluantsAtmosphériques à Transduction Optique et à base de Matériaux Nanoporeux. Application à la détection des Composés Carbonylés pour le Contrôle de la Qualité de l'Air, 2009, Université de Paris XI.

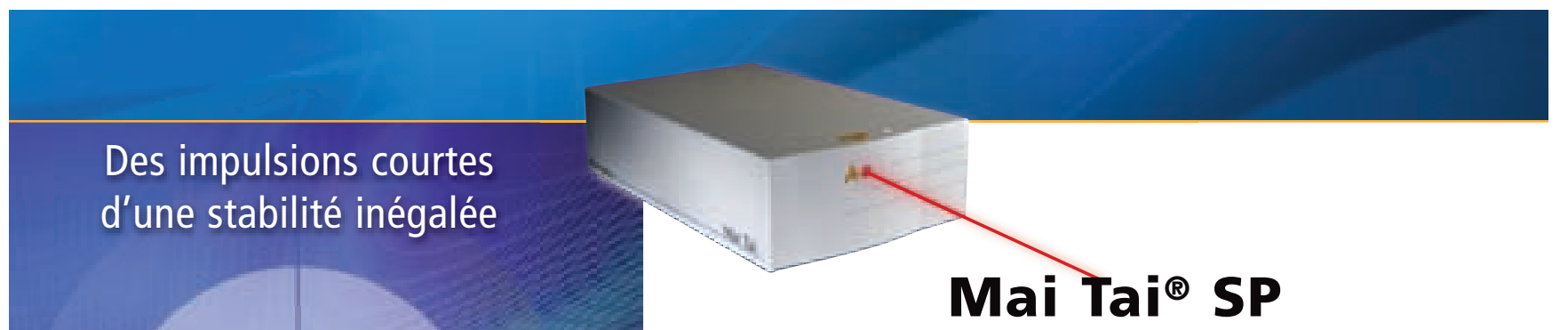

Le nouveau Mai Tai ${ }^{\circledR}$ SP est le premier oscillateur à impulsions courtes entièrement automatique et de parfaite stabilité pour I'injection d'amplificateur. Le laser Mai Tai SP produit des impulsions dont la largeur va de 100 fs à moins de 25 fs, sa largeur de bande est entièrement réglable par ordinateur. Notre technologie StabiLok ${ }^{\circledR}$ exclusive garantit que le pointage et la stabilité du faisceau sont constamment optimisés: ce laser se passe entièrement de réalignement, d'où une stabilité et une souplesse sans comparaison.

Largeur de bande réglable

\section{Pour des informations détaillées, \\ appelez-nous ou visitez www.newport.com/maitai-sp}

